Article

\title{
Joint Probabilistic-Nyquist Pulse Shaping for an LDPC-Coded 8-PAM Signal in DWDM Data Center Communications
}

\author{
Xiao Han ${ }^{1,2, *}$, Mingwei Yang ${ }^{1}$, Ivan B. Djordjevic ${ }^{1}$, Yang Yue ${ }^{2}$, Qiang Wang ${ }^{2}$, Zhen $\mathrm{Qu}^{2}$ and \\ Jon Anderson ${ }^{2}$ \\ 1 ECE Department, University of Arizona, Tucson, AZ 85721, USA; mingweiyang@email.arizona.edu (M.Y.); \\ ivan@email.arizona.edu (I.B.D.) \\ 2 Juniper Networks, 1133 Innovation Way, Sunnyvale, CA 94089, USA; yyue@juniper.net (Y.Y.); \\ qiwang.thresh@gmail.com (Q.W.); zqu@juniper.net (Z.Q.); jonanderson@juniper.net (J.A.) \\ * Correspondence: xhan322@email.arizona.edu
}

Received: 1 November 2019; Accepted: 18 November 2019; Published: 20 November 2019

\begin{abstract}
M-ary pulse-amplitude modulation (PAM) meets the requirements of data center communication because of its simplicity, but coarse entropy granularity cannot meet the dynamic bandwidth demands, and there is a large capacity gap between uniform formats and the Shannon limit. The dense wavelength division multiplexing (DWDM) system is widely used to increase the channel capacity, but low spectral efficiency of the intensity modulation/direct detection (IM/DD) solution restricts the throughput of the modern DWDM data center networks. Probabilistic shaping distribution is a good candidate to offer us a fine entropy granularity and efficiently reduce the gap to the Shannon limit, and Nyquist pulse shaping is widely used to increase the spectral efficiency. We aim toward the joint usage of probabilistic shaping and Nyquist pulse shaping with low-density parity-check (LDPC) coding to improve the bit error rate (BER) performance of 8-PAM signal transmission. We optimized the code rate of the LDPC code and compared different Nyquist pulse shaping parameters using simulations and experiments. We achieved a $0.43 \mathrm{~dB}$ gain using Nyquist pulse shaping, and a $1.1 \mathrm{~dB}$ gain using probabilistic shaping, while the joint use of probabilistic shaping and Nyquist pulse shaping achieved a $1.27 \mathrm{~dB}$ gain, which offers an excellent improvement without upgrading the transceivers.
\end{abstract}

Keywords: pulse amplitude modulation; nyquist pulse shaping; DWDM system; LDPC coding

\section{Introduction}

In view of the current development of the annual growth rate of data center transmission, the widely used coherent optical communication [1-5] is moving toward the data center networks market, but has not dominated because of its high cost, high power consumption, and implementation complexity. Self-coherent detection is a viable solution to reduce the cost, but its expensive coherent receiver still limits its use to a narrow range of applications [6-8]. To match the low-cost requirement, many researchers have paid increasing attention to pulse-amplitude modulation (PAM) [9-11]. However, for the currently widely used uniform distribution, coarse entropy granularity of M-ary PAM $(\mathrm{M}=2,4,8, \ldots)$ cannot meet the dynamic bandwidth demands. More importantly, there exists a large capacity gap between the uniform modulation formats and the Shannon limit.

To compensate for the performance loss, in recent years, a constellation shaping scheme has attracted increasing research attention, which include geometric shaping (GS) [12-17], probabilistic shaping (PS) [18-23], and hybrid geometric-probabilistic shaping [24-27]. These different shaping 
schemes can approach the Shannon limit. Since it may be easier to have a common standard agreement among network service providers if the PS scheme is used for constellation shaping, PS should be more suitable to be used in data center networks. PS can be realized using Huffman coding [28,29], many-to-one mapping [30], and a constant composition distribution matcher (CCDM) [31,32]. Given that a CCDM can flexibly generate fractional entropy without systematic error, the optical industry is more inclined to apply a CCDM-based PS scheme. PS can not only be used for quadrature amplitude modulation (QAM) formats, but also for a PAM scheme [33-35]. For a PAM scheme, it imposes an exponential-like distribution on a set of equidistant constellation points. It transmits symbols with smaller amplitudes more often than larger ones, which can offer us a fine entropy granularity and enable a transmission with a lower signal-to-noise ratio (SNR) at the same forward error correction (FEC) overhead.

The requirement of channel capacity is greatly increasing nowadays, which leads to the dense wavelength division multiplexing (DWDM) system being widely using in data center networks [36-38], but the low spectral efficiency of the intensity modulation/direct detection (IM/DD) solution restricts the throughput of the modern DWDM data center networks, which results in large investments to upgrade the transceivers in order to meet the increasing bandwidth requirement.

Nyquist pulse shaping (NPS) is a good solution to increase the spectral efficiency of a DWDM system [39,40]; it uses a raised-cosine filter to limit the effective bandwidth and can reduce the inter-symbol interference (ISI) by properly selecting the roll-off factor (ROF) for NPS.

The purpose of this paper was to demonstrate the joint usage of both the PS distribution and NPS in a DWDM system for short-reach applications, in particular data center networks. In addition to the joint shaping format, the employment of a suitable FEC code was also important for improving the overall performance. Low-density parity-check (LDPC) codes represent excellent FEC candidates to be applied together with the proposed shaping scheme. We transmitted LDPC-coded 8-PAM signals for both uniform and nonuniform signaling and compared the performance for different LDPC code rates. We further evaluated the performance improvements when NPS was used for different ROFs in both PS and uniform distribution-based systems. We experimentally evaluated the bit error rate (BER) performance improvement of the proposed joint shaping scheme, compared with the uniform signaling scheme.

The rest of the paper is organized as follows. In Section 2, we introduce the DWDM system employing the proposed joint probabilistic-Nyquist pulse shaping scheme. In Section 3, we demonstrate the improvements with respect to uniform signaling via simulation and experimental verifications. Relevant concluding remarks are provided in Section 4.

\section{Proposed PS-NPS-8-PAM-Based DWDM System}

Figure 1 shows the proposed LDPC-coded PS-NPS-8-PAM signal generator. The input was a pseudorandom binary sequence (PRBS), and after the distribution matcher (DM), there was an array of different amplitudes that satisfy a certain probability distribution. After binary labelling and LDPC encoding [36], we obtained a block of LDPC-coded binary bits. After mapping to the PAM constellation points, we performed the Nyquist pulse shaping, followed by the DWDM multiplexing.

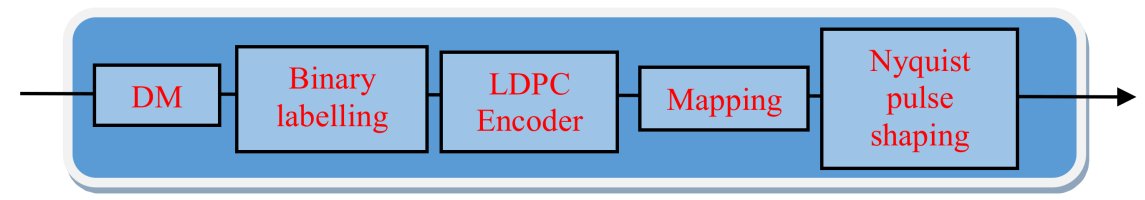

Figure 1. Low-density parity-check (LDPC)-coded, probabilistic shaping, Nyquist pulse shaping, 8-level pulse-amplitude modulation (PS-NPS-8-PAM) signal generator. DM: Distribution matcher. 
The PS distribution performances are highly dependent on a selected distribution function. In this paper, we used an exponential distribution [33], in which different constellation points $a_{i}$ were transmitted with probabilities determined using:

$$
\mathrm{P}\left(\mathrm{a}_{\mathrm{i}}\right)=\exp \left(-\lambda\left\|\mathrm{a}_{\mathrm{i}}\right\|\right) / \mathrm{Z}(\lambda), \lambda \geq 0,
$$

where $Z(\lambda)$ is the normalization function used to ensure that the probabilities of occurrence of symbols sum up to one, which means $Z(\lambda)$ is defined as:

$$
\mathrm{Z}(\lambda)=\sum_{\mathrm{i}} \exp \left(-\lambda\left\|\mathrm{a}_{\mathrm{i}}\right\|\right)
$$

Nyquist pulse shaping is widely used in DWDM systems to improve the spectral efficiency [40]. It often uses a low-pass raised-cosine (RC) filter with the frequency response being:

$$
\mathrm{H}_{\mathrm{RC}}(\omega)=\left\{\begin{array}{cc}
\mathrm{T}_{\mathrm{S}} & 0 \leq|\omega|<\pi(1-\mathrm{ROF}) / \mathrm{T}_{\mathrm{S}} \\
\frac{\mathrm{T}_{\mathrm{S}}}{2}\left(1-\sin \left[\frac{\mathrm{T}_{\mathrm{S}}}{2 \times \mathrm{ROF}}\left(|\omega|-\frac{\pi}{\mathrm{T}_{\mathrm{S}}}\right)\right]\right), & \pi(1-\mathrm{ROF}) / \mathrm{T}_{\mathrm{S}} \leq|\omega|<\pi(1+\mathrm{ROF}) / \mathrm{T}_{\mathrm{S}} \\
0 & |\omega|>\pi(1+\mathrm{ROF}) / \mathrm{T}_{\mathrm{S}}
\end{array}\right.
$$

where $\omega$ is the angular frequency, $T_{s}$ is the symbol duration, and ROF is the roll-off factor mentioned before. A small ROF value can make the frequency response an almost rectangular shape and be able to reduce the channel spacing but comes with a longer memory length and a higher generation complexity. In this study, we used the square-root raised cosine (SRRC) filter, whose transfer function is given as:

$$
\mathrm{H}_{\text {SRRC }}(\omega)=\sqrt{\mathrm{H}_{\mathrm{RC}}(\omega)}
$$

Figure 2 shows the frequency response for two different ROF values of neighboring DWDM channels, with the channel spacing set to $50 \mathrm{GHz}$. It is clear that a smaller ROF had a better spectral efficiency with a lower inter-channel crosstalk.

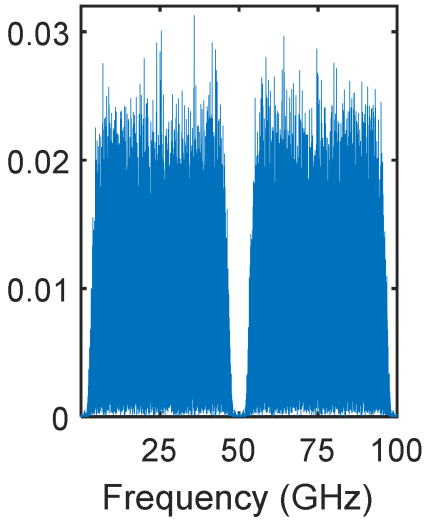

(a)

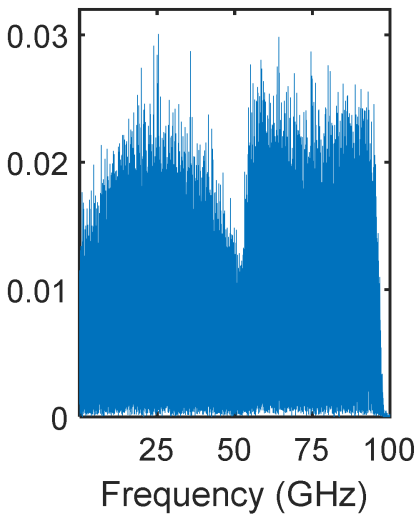

(b)

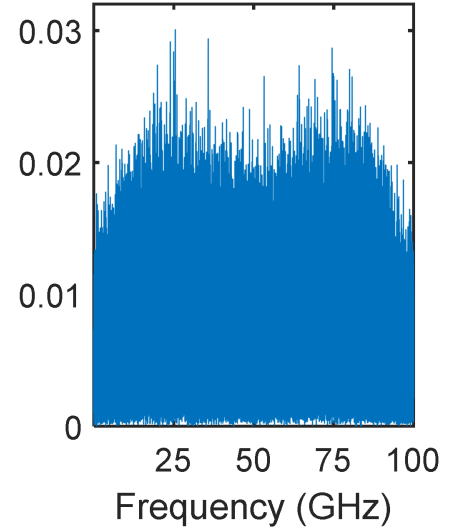

(c)

Figure 2. Frequency response with different roll-off factors (ROFs): (a) $\mathrm{ROF}_{1}=\mathrm{ROF}_{2}=0.1 ;(\mathbf{b}) \mathrm{ROF}_{1}=1$, $\mathrm{ROF}_{2}=0.1 ;$ and $(\mathbf{c}) \mathrm{ROF}_{1}=\mathrm{ROF}_{2}=1$.

Figure 3 shows the whole DWDM system. On the transmitter side, we generated the PS-NPS-8-PAM signals using the generator in Figure 1 and sent them to the modulator for each channel with a different frequency. Then, after transmission, at the receiver, we used a super Gaussian filter to select every target frequency (wavelength). 


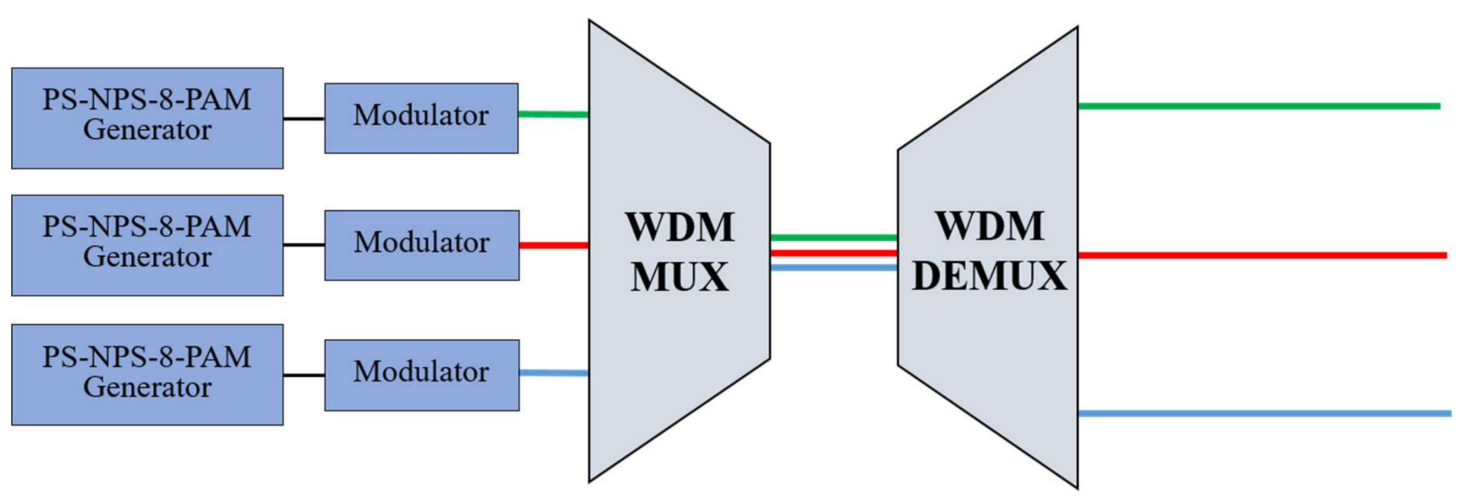

Figure 3. Structure of the PS-NPS-8-PAM-based DWDM system. DEMUX: demultiplexer.

\section{Simulation and Data Center Experimental Results}

In this section, we first describe the optimization of the code rate of the LDPC encoder for the PS distribution and show a different ROF factor performance using simulations. Then, we introduce the experimental setup and the improvement we achieved from our PS-NPS-8-PAM scheme.

\subsection{Simulation Results}

Figure 4 shows the BER performance comparison for LDPC-coded 8-PAM signals with PS and uniform distributions for different LDPC code rates. As the PS distribution has a smaller entropy, we needed to make sure that each modulation scheme had the same information rate to guarantee that the comparison was fair; in other words, they had the same FEC overhead. The code rate of the uniform distribution was 0.6 , so the information rate was 1.8 bits/symbol, with a $66.7 \%$ FEC overhead. From Figure 4, we can see that all PS distributions outperformed the uniform distribution, and the best improvement offered a $0.8 \mathrm{~dB}$ signal-to-noise ratio (SNR) gain over the uniform distribution at a BER of $10^{-5}$, which appeared when code rate ( $r$ ) equal to 0.8 .

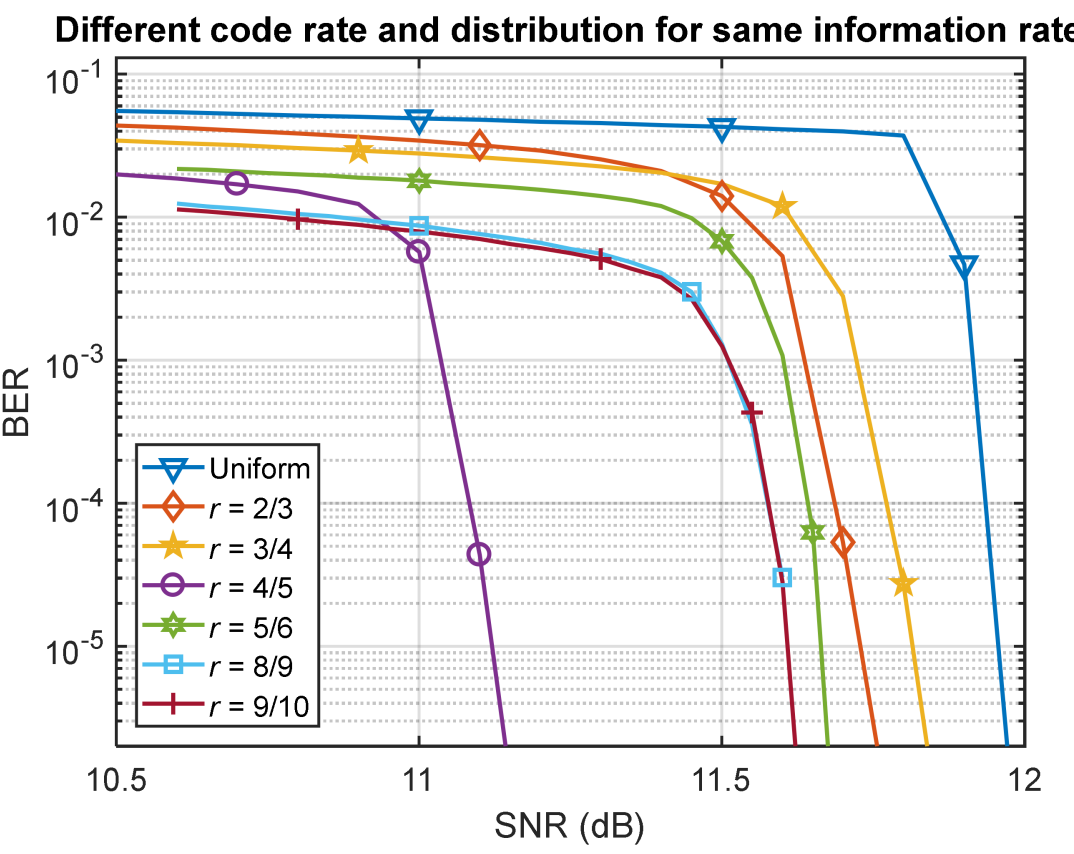

Figure 4. Bit error ratio (BER) performance for different LDPC code rates $(r)$. SNR: Signal-to-noise ratio.

Figure 5 shows the BER performance of the LDPC-coded PS and uniform distribution for different values of the ROF. We can see for both distribution schemes that a smaller ROF achieved a better 
BER performance, which was caused by the reason shown in Figure 2: a smaller ROF value can lead to the frequency response of a rectangular shape, improving the spectral efficiency. For every ROF value considered, we found that the PS distribution-based scheme always outperformed uniformly distribution-based one.

Figure 5a shows a very clear error flow when the ROF was set to 0.5. Namely, every LDPC code had an effective SNR region, in which it sufficiently improved the BER performance after a corresponding SNR threshold. According to Figure 2, a larger ROF brought a wider bandwidth and more inter-channel crosstalk, which prevented us reaching the SNR threshold of the employed LDPC code.

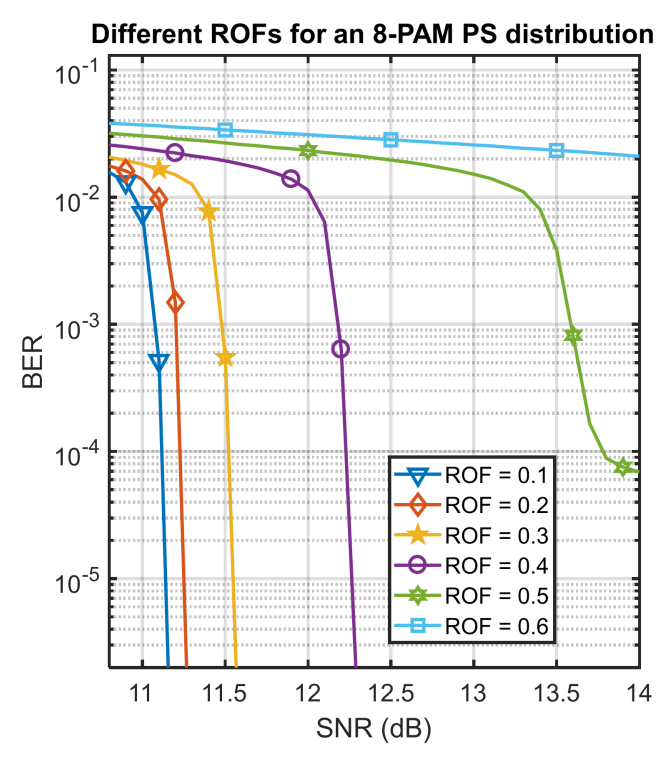

(a)

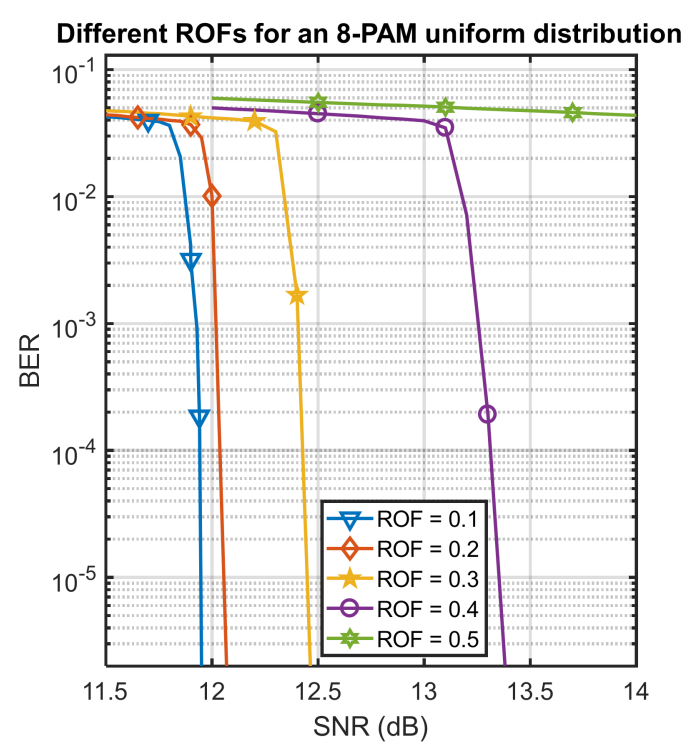

(b)

Figure 5. BER performance for different ROF values for: (a) a PS distribution with an LDPC code of rate $r=0.8$ and (b) a uniform distribution with an LDPC code of rate $r=0.6$.

\subsection{Experimental Setup and Results}

The performance of the LDPC-coded PS-NPS-8-PAM transmission in a fiber channel-based DWDM system was experimentally verified with the testbed depicted in Figure 6 . Three $10 \mathrm{kHz}$-linewidth, continuous-wave, tunable sources (with the following center frequencies: $f_{1}=193.30 \mathrm{THz}, f_{2}=$ $193.35 \mathrm{THz}$, and $f_{3}=193.40 \mathrm{THz}$ ) were coupled by an optical coupler and launched to a Mach-Zehnder modulator. The PRBS was sent to our LDPC-coded PS-NPS-8-PAM generator. Then, the LDPC-coded PS-NPS signals were sent to an arbitrary waveform generator (AWGen) to create 11.5 GBaud 8-PAM signals with a $66.7 \%$ FEC overhead, which meant the bit rate was $20.7 \mathrm{Gbps}$. After being converted to the optical domain by the modulator, the resulting signals were boosted by an erbium-doped fiber amplifier (EDFA) with a $6 \mathrm{~dB}$ noise figure. The enhanced signal was then sent to a $1 \times 3$ coupler to be split and interleaved into three fibers with different lengths (delay lines). The corresponding outputs were then applied to a $32 \times 32$ arrayed waveguide grating (AWG)-based datacenter network at three different input ports. Every input port worked as a selective bandpass filter. At the output port, we obtained three different center frequencies with different delays, which worked as our DWDM system. The output signal was then mixed with an amplified spontaneous emission (ASE) noise signal using a $2 \times 2$ coupler. We also employed a variable optical attenuator (VOA) after the ASE noise source to emulate the different optical SNR (OSNR) channel conditions. At the receiver side, the targeted frequency $f_{2}$ was selected by a tunable filter (TF) and detected using a photodetector (PD). To collect the received baseband signal, we employed a $100 \mathrm{GS} / \mathrm{s}$ digital phosphor oscilloscope from Tektronix. Then, we performed offline digital signal processing (DSP) with the collected signals. 


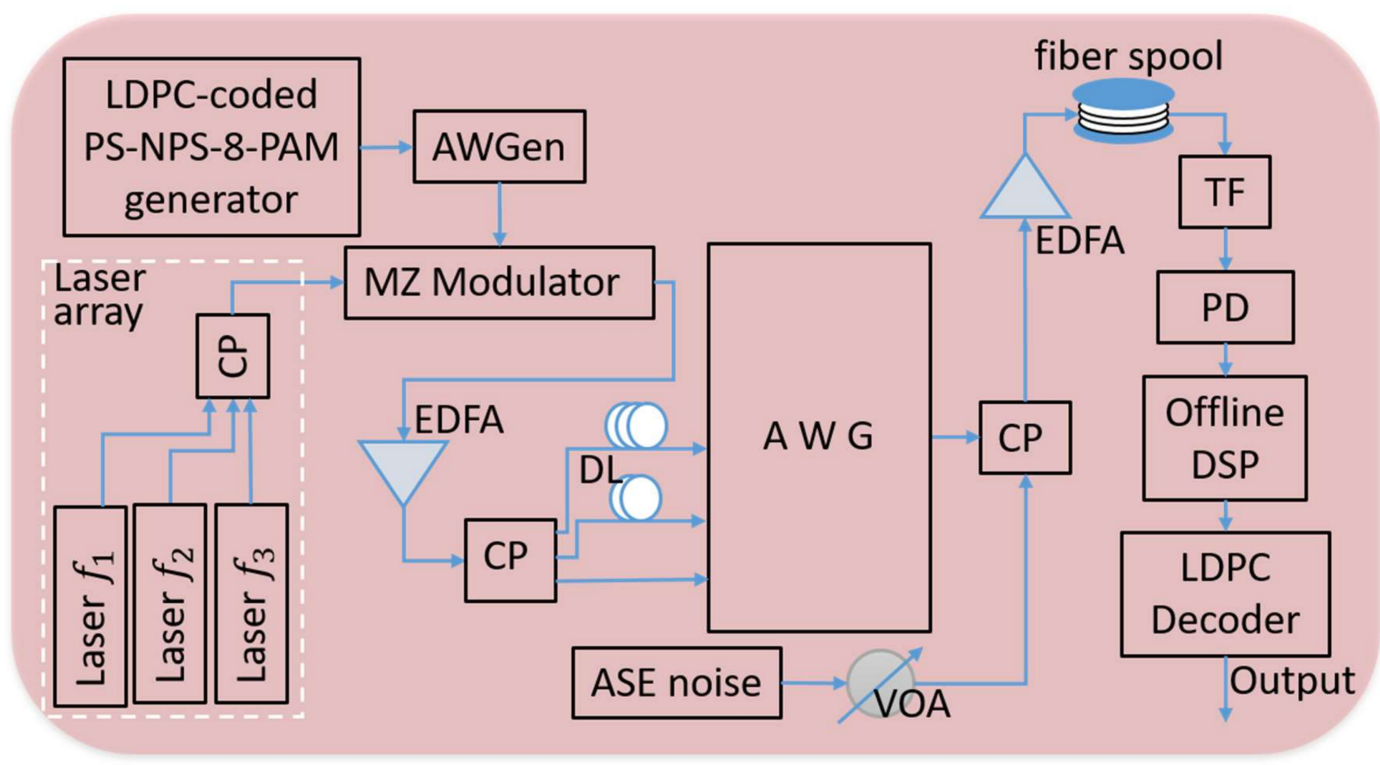

Figure 6. Experimental data center setup. CP: coupler, DL: delay line.

The BER performance of the LDPC-coded PS-NPS-8-PAM is shown in Figure 7a. We transmitted the PS-NPS-8-PAM signals with ROF $=0.8$ and 1 , and compared them against a PS-8-PAM. From this figure, we can see that when $\mathrm{ROF}=1$, it performed the same as the PS-8-PAM without NPS, which is because ROF = 1 will not change the pulse shape. On the other hand, when we set the ROF to 0.8 , we obtained a $0.43 \mathrm{~dB}$ shaping gain improvement in OSNR at BER $=10^{-5}$. In Figure $7 \mathrm{~b}$, we compare the LDPC-coded PS- and uniform distribution-based schemes with both ROFs set to 0.8. We can see that uniform distribution required higher OSNRs, and we obtained a $1.1 \mathrm{~dB}$ shaping gain improvement in OSNR at BER $=10^{-5}$.

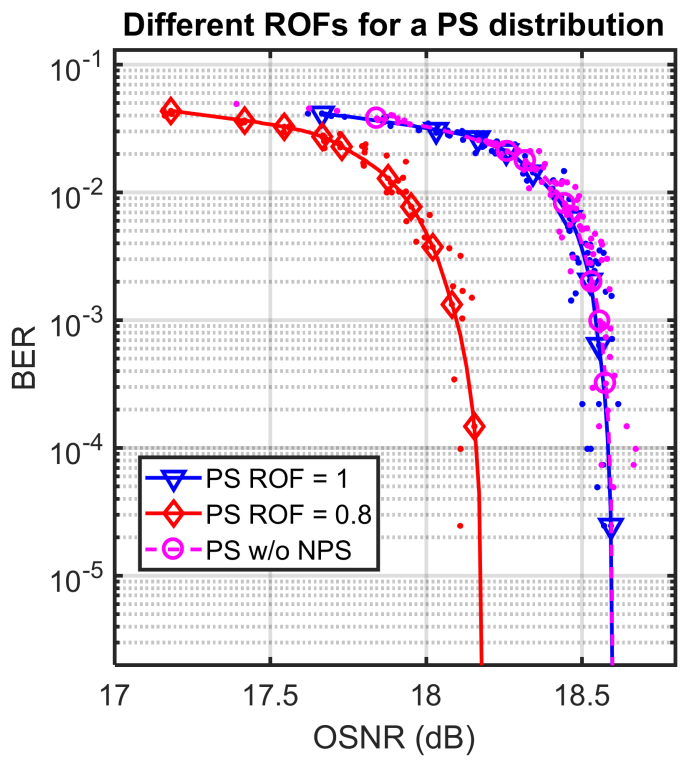

(a)

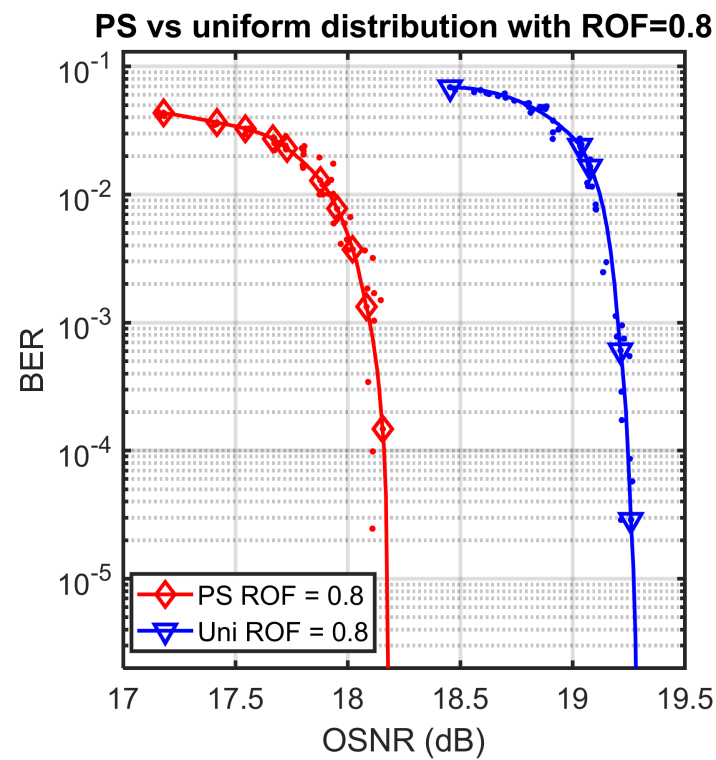

(b)

Figure 7. BER performance for: (a) a PS distribution with different ROFs and without NPS, and (b) a PS and uniform distribution comparison with the same ROF. OSNR: optical signal-to-noise ratio.

Figure 8 shows the comparison of BER performance between the LDPC-coded PS-NPS-8-PAM and LDPC-coded uniform distributed 8-PAM schemes. Evidently, the joint usage of PS and NPS could obtain a $1.27 \mathrm{~dB}$ OSNR improvement at $\mathrm{BER}=10^{-5}$ compared to the uniform signaling. 


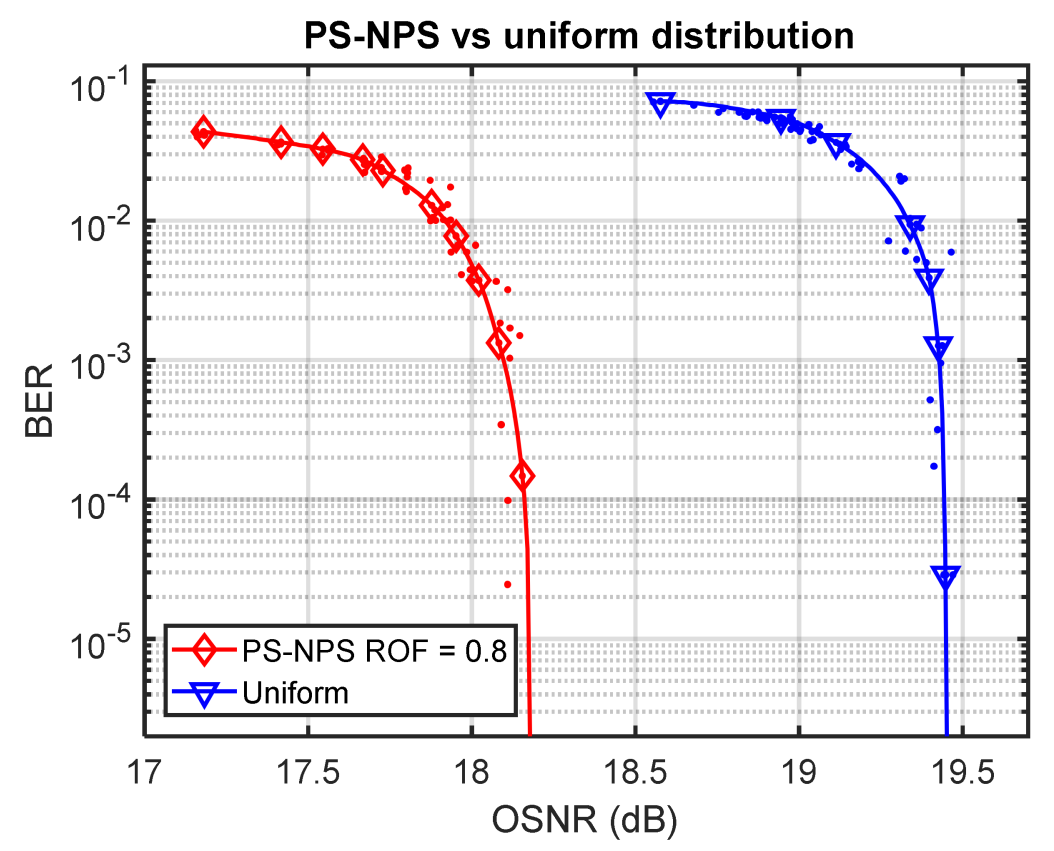

Figure 8. BER performance of an LDPC-coded PS-NPS-8-PAM against an LDPC-coded uniform 8-PAM.

\section{Conclusions}

In our simulation results, we found that a smaller ROF could provide a better BER performance, and to optimize the PS performance, we needed to consider both the signal entropy and the error correction capability of an LDPC code. In practical usage, there will be many potential limits on the ROF and FEC overhead, since in most cases, we can only use an appropriate ROF for a certain FEC overhead. We have shown that the optimization was not highly sensitive to either the ROF or FEC overhead, and we could do the optimization on any limit of the ROF and FEC overhead. The improvement can be achieved without any equipment upgrade, which means that the proposed LDPC-coded PS-NPS-8-PAM scheme can be widely applied in data center communications and other short-reach applications. We have shown that the Nyquist pulse shaping could provide a $0.43 \mathrm{~dB}$ improvement, and the probabilistic shaping provided a $1.1 \mathrm{~dB}$ gain. By the joint use of probabilistic shaping and Nyquist pulse shaping, we obtained a $1.27 \mathrm{~dB}$ performance gain.

Author Contributions: This research was conducted by X.H. M.Y. offered experimental support. Y.Y., Q.W., Z.Q. and J.A. supervised the NPS and PS idea and simulation during Han's internship at Juniper Networks. Both the simulation and the experimental demonstration were supervised by I.B.D.

Funding: This research was funded by Juniper Networks and NSF.

Conflicts of Interest: The authors declare no conflict of interest.

\section{References}

1. Huang, M.F.; Tanaka, A.; Ip, E.; Huang, Y.K.; Qian, D.; Zhang, Y.; Zhang, S.; Ji, P.N.; Djordjevic, I.B.; Wang, T.; et al. Terabit/s Nyquist superchannels in high capacity fiber field trials using DP-16QAM and DP-8QAM modulation formats. J. Lightwave Technol. 2014, 32, 776-782. [CrossRef]

2. Perin, J.K.; Shastri, A.; Kahn, J.M. Design of Low-Power DSP-Free Coherent Receivers for Data Center Links. J. Lightwave Technol. 2017, 35, 4650-4662. [CrossRef]

3. Cai, J.X.; Batshon, H.G.; Mazurczyk, M.; Zhang, H.; Sun, Y.; Sinkin, O.V.; Foursa, D.; Pilipetskii, A.N. 64QAM based coded modulation transmission over transoceanic distance with $>60 \mathrm{~Tb} / \mathrm{s}$ capacity. In Proceedings of the Optical Fiber Communication Conference (OFC), Los Angeles, CA, USA, 22-26 March 2015.

4. Qu, Z.; Li, Y.; Mo, W.; Yang, M.; Zhu, S.; Kilper, D.; Djordjevic, I.B. Performance optimization of PM-16QAM transmission system enabled by real-time self-adaptive coding. Opt. Lett. 2017, 42, 4211-4214. [CrossRef] 
5. Winzer, P.J.; Neilson, D.T.; Chraplyvy, A.R. Fiber-optic transmission and networking: The previous 20 and the next 20 years. Opt. Express 2018, 26, 24190-24239. [CrossRef]

6. Puttnam, B.J.; Luis, R.; Delgado-Mendinueta, J.-M.; Sakaguchi, J.; Klaus, W.; Awaji, Y.; Wada, N.; Kanno, A.; Kawanishi, T. High-capacity self-homodyne PDM-WDM-SDM transmission in a 19-core fiber. Opt. Express 2014, 22, 21185-21191. [CrossRef]

7. Qu, Z.; Fu, S.; Zhang, M.; Tang, M.; Shum, P.; Liu, D. Analytical investigation on self-homodyne coherent system based on few-mode fiber. IEEE Photonics Technol. Lett. 2014, 26, 74-77. [CrossRef]

8. Puttnam, B.J.; Luís, R.S.; Mendinueta, J.M.D.; Sakaguchi, J.; Klaus, W.; Kamio, Y.; Nakamura, M.; Wada, N.; Awaji, Y.; Kanno, A.; et al. Self-homodyne detection in optical communication systems. Photonics 2014, 1, 110-130. [CrossRef]

9. Zhong, K.; Zhou, X.; Gao, Y.; Chen, W.; Man, J.; Zeng, L.; Lau, A.P.T.; Lu, C. 140-Gb/s 20-km Transmission of PAM-4 Signal at $1.3 \mu \mathrm{m}$ for Short Reach Communications. IEEE Photonics Technol. Lett. 2015, 27, 1757-1760. [CrossRef]

10. Eiselt, N.; Wei, J.; Griesser, H.; Dochhan, A.; Eiselt, M.; Elbers, J.-P.; Olmos, J.J.V.; Monroy, I.T. First real-time 400G PAM-4 demonstration for inter-data center transmission over $100 \mathrm{~km}$ of SSMF at $1550 \mathrm{~nm}$. In Proceedings of the Optical Fiber Communications Conference and Exhibition (OFC), Anaheim, CA, USA, 20-24 March 2016.

11. Mestre, M.A.; Mardoyan, H.; Konczykowska, A.; Rios-Müller, R.; Renaudier, J.; Jorge, F.; Duval, B.; Dupuy, J.-Y.; Ghazisaeidi, A.; Jennevé, P.; et al. Direct detection transceiver at 150-Gbit/s net data rate using PAM 8 for optical interconnects. In Proceedings of the 2015 European Conference on Optical Communication (ECOC), Valencia, Spain, 27 September-1 October 2015.

12. Chen, B.; Okonkwo, C.; Hafermann, H.; Alvarado, A. Increasing achievable information rates via geometric shaping. In Proceedings of the European Conference on Optical Communication (ECOC), Rome, Italy, 23-27 September 2018.

13. Qu, Z.; Djordjevic, I.B.; Anderson, J. Two-Dimensional Constellation Shaping in Fiber-Optic Communications. Appl. Sci. 2019, 9, 1889. [CrossRef]

14. Khandani, A.K.; Kabal, P. Shaping multidimensional signal spaces. I. Optimum shaping, shell mapping. IEEE Trans. Inf. Theory 1993, 39, 1799-1808. [CrossRef]

15. Qu, Z.; Djordjevic, I.B. Geometrically shaped 16QAM outperforming probabilistically shaped 16QAM. In Proceedings of the European Conference on Optical Communication (ECOC), Gothenburg, Sweden, 17-21 September 2017.

16. Forney, G.D. Trellis shaping. IEEE Trans. Inf. Theory 1992, 38, 281-300. [CrossRef]

17. Forney, G.D.; Wei, L.-F. Multidimensional constellations-Part I: Introduction, figures of merit, and generalized cross constellations. IEEE J. Sel. Areas Commun. 1989, 7, 877-892. [CrossRef]

18. Cho, J.; Chen, X.; Chandrasekhar, S.; Raybon, G.; Dar, R.; Schmalen, L.; Burrows, E.; Adamiecki, A.; Corteselli, S.; Pan, Y.; et al. Trans-atlantic field trial using high spectral efficiency probabilistically shaped 64-QAM and single-carrier real-time 250-Gb/s 16-QAM. J. Lightware Technol. 2018, 36, 103-113. [CrossRef]

19. Calderbank, A.R.; Ozarow, L.H. Non-equiprobable signaling on the Gaussian channel. IEEE Trans. Inf. Theory 1990, 36, 726-740. [CrossRef]

20. Qu, Z.; Djordjevic, I.B. On the Probabilistic Shaping and Geometric Shaping in Optical Communication Systems. IEEE Access 2019, 7, 21454-21464. [CrossRef]

21. Steiner, F.; Böcherer, G. Comparison of Geometric and Probabilistic Shaping with Application to ATSC 3.0. In Proceedings of the International ITG Conference on Systems, Communications and Coding (SCC), Hamburg, Germany, 6-9 February 2017.

22. Fehenberger, T.; Alvarado, A.; Bocherer, G.; Hanik, N. On probabilistic shaping of quadrature amplitude modulation for the nonlinear fiber channel. J. Lightware Technol. 2016, 34, 5063-5073. [CrossRef]

23. Böcherer, G.; Steiner, F.; Schulte, P. Bandwidth efficient and rate-matched low-density parity-check coded modulation. IEEE Trans. Commun. 2015, 63, 4651-4665. [CrossRef]

24. Qu, Z.; Djordjevic, I.B. Hybrid Probabilistic-Geometric Shaping in Optical Communication Systems. In Proceedings of the IEEE Photonics Conference (IPC), Reston, VA, USA, 30 September-4 October 2018. 
25. Batshon, H.G.; Mazurczyk, M.V.; Cai,J.X.; Sinkin, O.V.; Paskov, M.; Davidson, C.R.; Wang, D.; Bolshtyansky, M.; Foursa, D. Coded modulation based on 56APSK with hybrid shaping for high spectral efficiency transmission. In Proceedings of the 2017 European Conference on Optical Communication (ECOC), Gothenburg, Sweden, 17-21 September 2017.

26. Ren, J.; Liu, B.; Xu, X.; Zhang, L.; Mao, Y.; Wu, X.; Zhang, Y.; Jiang, L.; Xin, X. A probabilistically shaped star-CAP-16/32 modulation based on constellation design with honeycomb-like decision regions. Opt. Express 2019, 27, 2732-2746. [CrossRef]

27. Han, X.; Yang, M.; Djordjevic, I.B. Hybrid Probabilistic-Geometric-Shaped 8-PAM Suitable for Data Centers' Communication. In Proceedings of the Asia Communications and Photonics Conference (ACP), Hangzhou, China, 26-29 October 2018.

28. Qu, Z.; Lin, C.; Liu, T.; Djordjevic, I.B. Experimental study of nonlinearity tolerant modulation formats based on LDPC coded non-uniform signaling. In Proceedings of the Optical Fiber Communications Conference and Exhibition (OFC), Los Angeles, CA, USA, 19-23 March 2017.

29. Yankovn, M.P.; Ros, F.D.; Silva, E.P.; Forchhammer, S.; Larsen, K.J.; Oxenløwe, L.K.; Galili, M.; Zibar, D. Constellation Shaping for WDM Systems Using 256QAM/1024QAM with Probabilistic Optimization. J. Lightware Technol. 2016, 34, 5146-5156. [CrossRef]

30. Gallager, R.G. Information Theory and Reliable Communication; Wiley: Hoboken, NJ, USA, 1968.

31. Schulte, P.; Bocherer, G. Constant composition distribution matching. IEEE Trans. Inf. Theory 2016, 62, 430-434. [CrossRef]

32. Fehenberger, T.; Millar, D.S.; Koike-Akino, T.; Kojima, K.; Parsons, K. Multiset-partition distribution matching. IEEE Trans. Commun. 2018, 67, 1885-1893. [CrossRef]

33. Han, X.; Djordjevic, I.B. Probabilistically shaped 8-PAM suitable for data centers communication. In Proceedings of the International Conference on Transparent Optical Networks (ICTON), Bucharest, Romania, 1-5 July 2018.

34. Eriksson, T.A.; Chagnon, M.; Buchali, F.; Schuh, K.; Brink, S.T.; Schmalen, L. 56 Gbaud Probabilistically Shaped PAM8 for Data Center Interconnects. In Proceedings of the 2017 European Conference on Optical Communication (ECOC), Gothenburg, Sweden, 17-21 September 2017.

35. Sun, L.; Wang, C.; Du, J.; Liang, C.; Zhang, W.; Xu, K.; Zhang, F.; He, Z. Dyadic Probabilistic Shaping of PAM-4 and PAM-8 for Cost-Effective VCSEL-MMF Optical Interconnection. IEEE Photonics J. 2019, 11, 1-12. [CrossRef]

36. Lu, P.; Zhang, L.; Liu, X.; Yao, J.; Zhu, Z. Highly efficient data migration and backup for big data applications in elastic optical inter-data-center networks. IEEE Netw. 2015, 29, 36-42. [CrossRef]

37. Mahimkar, A.; Chiu, A.; Doverspike, R.; Feuer, M.D.; Magill, P.; Mavrogiorgis, E.; Pastor, J.; Woodward, S.L.; Yates, J. Bandwidth on Demand for Inter-Data Center Communication. In Proceedings of the 10th ACM Workshop on Hot Topics in Networks (HotNets-X'11), New York, NY, USA, 14-15 November 2011; ACM: New York, NY, USA, 2011.

38. Chen, K.; Singla, A.; Singh, A.; Ramachandran, K.; Xu, L.; Zhang, Y.; Wen, X.; Chen, Y. OSA: An Optical Switching Architecture for Data Center Networks with Unprecedented Flexibility. IEEE/ACM Trans. Netw. 2014, 22, 498-511. [CrossRef]

39. Hirai, R.; Kikuchi, N.; Fukui, T. High-spectral efficiency DWDM transmission of 100-Gbit/s/lambda IM/DD single sideband-baseband-Nyquist-PAM8 signals. In Proceedings of the Optical Fiber Communications Conference and Exhibition (OFC), Los Angeles, CA, USA, 19-23 March 2017.

40. Yue, Y.; Wang, Q.; Anderson, J. Transmitter skew tolerance and spectral efficiency tradeoff in high baud-rate QAM optical communication systems. Opt. Express 2018, 26, 15045-15058. [CrossRef]

(C) 2019 by the authors. Licensee MDPI, Basel, Switzerland. This article is an open access article distributed under the terms and conditions of the Creative Commons Attribution (CC BY) license (http://creativecommons.org/licenses/by/4.0/). 